\title{
CSF Based Non-ground Points Extraction From LiDAR Data
}

\author{
Aojie Shen ${ }^{\mathrm{a}}$, Wuming Zhang ${ }^{\mathrm{b}}$ *, Huiling Shi ${ }^{\mathrm{c}}$ \\ a,b,c State Key Laboratory of Remote Sensing Science, Institute of Remote Sensing Science and Engineering, Faculty of Geographical \\ Science, Beijing Normal University, Beijing 100875, China (wumingz@bnu.edu.cn)
}

KEY WORDS: region growing; seed point; CSF; non-ground point; cloth resolution

\begin{abstract}
:
Region growing is a classical method of point cloud segmentation. Based on the idea of collecting the pixels with similar properties to form regions, region growing is widely used in many fields such as medicine, forestry and remote sensing. In this algorithm, there are two core problems. One is the selection of seed points, the other is the setting of the growth constraints, in which the selection of the seed points is the foundation. In this paper, we propose a CSF (Cloth Simulation Filtering) based method to extract the non-ground seed points effectively. The experiments have shown that this method can obtain a group of seed spots compared with the traditional methods. It is a new attempt to extract seed points.
\end{abstract}

\section{INTRODUCTION}

Originally proposed by Sun and Salvaggio, region growing refers to the process of developing groups of pixels or regions into larger areas (Sun S, 2013). Ideas are starting from the collection of seed points (selected arrays) and gradually finding the pixels that are close to the seed points which mean their attributes match, their gray levels are similar, and their texture colors are the same. Then merged the pixels into the region (Zhengping Hu, 2007). Region growing as an excellent image segmentation algorithm is used in medicine (Jianfeng Lu, 2005), agriculture and other fields.

Recently, with the development of research, region growing has been applied from the two-dimensional image segmentation into the three-dimensional point cloud processing, especially the city non-ground point extraction. Yongjun Wang applied the area growing into the extraction of the roof of buildings. By selecting the seed point, the plane area growth and smoothing the surface, extract the smooth roof plane, laying the foundation for the reconstruction of the building. The method is traditional, that is: first calculate the normal vector and the curvature value of the point cloud; then choose the point with the smallest curvature as the seed point; and then begin region growing in its neighborhood; points whose difference between the normal vector and seed the points within the threshold are attributed to the group of seed points. The final integrity and correctness of reconstruction of the roof plane of the were about $90 \%$ and $95 \%$ (Wang Y, 2016).

Application of region growing in the image faces with several thorny problems: the problem of the selection of the seed points of the image, the problem of growth conditions, and the problem of stopping the growth conditions (Hui Wang, 2016). From the application situation, it is obvious that this problem still exists when region growing is applied to the three-dimensional point cloud and the seed point selection is more fundamental for this problem.

How to select representative seeds point is critical to the region growing method, and the result of the region growing algorithm depends largely on the selection of seed point. The existing seed point selection method has many problems such as too many seed points, low efficiency and insufficient non-ground minutia seed points. All along, researchers have looked for a robust way to extract the seed points. In order to solve the problem of seed point selection, a method of selecting the region growing seed point based on one-dimensional spectral difference is proposed (Xiuxia Li, 2016). The source images are pre-processed using non-linear mapping method and the target region in the liver is selected by man-machine interaction; Quasi-Monte Carlo's method is used for generating low-dispersion sequences points in the target region and the optical seed points are selected by calculating these points to improve the accuracy of the medical image segmentation and weaken the influence of selecting seed 


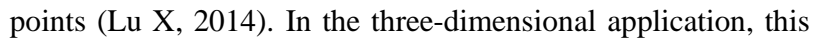
problem still needs to be resolved. M. Rook used region growing for automatic semantic annotation of 3D city models. Seed points is determined based on topological relations (Rook M, 2016). But in these situations where region growing is not robust, only isolated seed spots are extracted. Researchers are also trying to find a way to get groups of seed points. Anh-Vu Vo regarded a voxel as a point which is equivalent to a very small cube in space, so it has a lot of details that can be described separately. The main thing here is not the seed points, but the seed voxels. And then he carried out two steps. The first step was rough segmentation involved in completing the point cloud voxelization, calculating the voxel characteristics and then gathering it. The second step was refining. He will put the missing voxels according to the characteristics of the internal point to the corresponding surface and then achieve refining (Vo A V, 2015). Although the selection of the size of voxels in this method is still a problem and is greatly influenced by the data, Anh- $\mathrm{Vu}$ Vo roughly extracted a class of seed points. This paper presents a method to extract non-ground seed points using CSF (cloth simulation filter). It is a new attempt to extract seed points and has successfully extracted a group of non-ground seed points.

\section{METHODOLOGY}

In the region growing algorithm, the extraction of seed points is both the core and the foundation. This paper presents a method of extracting seed points from non-ground points with CSF and the potential and feasibility of this method for extracting nonground seed spots are illustrated by experiments.

\subsection{Non - ground Points Extraction Based on CSF}

CSF presented by Wuming Zhang (Zhang W, 2016), the basic idea is to reverse the point cloud, and then cover it with a cloth. Their research team analyzed the relationship between the cloth node and the point cloud, resulted in an approximate ground and finally extracted the ground point. The original intention of the method is to carry out the ground filter. Compared with the traditional algorithm which needs to set a large number of parameters to obtain high-precision, CSF only needs to set the cloth resolution, the number of iterations and classification threshold and other few parameters, and then it can extract ground points.

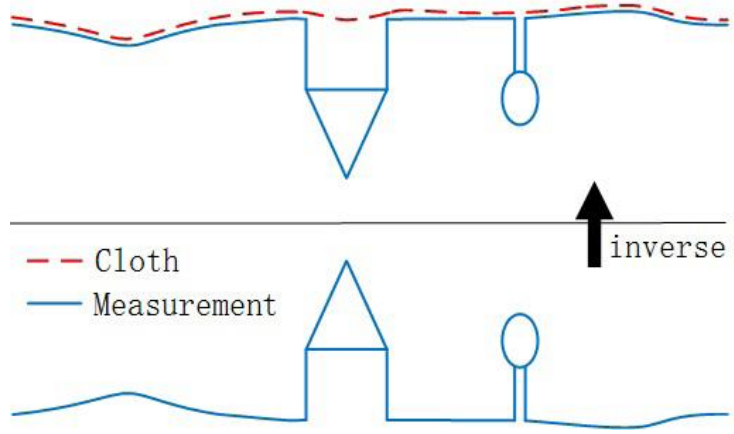

Figure 1. An overview of the cloth simulation algorithm

According to their research, they can get the terrain after the point cloud be inverted. Therefore, it is possible to get nonground points by not inverting the point cloud. The idea of this article is not to put the point cloud upside down, get high point cloud directly from the top cover, and this part is the seed points of the non-ground points.

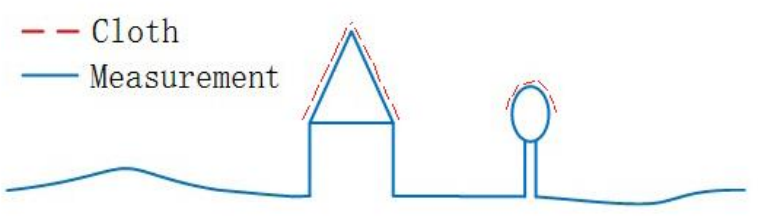

Figure 2. An overview of the cloth simulation algorithm(not inverting the point cloud)

This paper uses the data of cloud points from different areas which can be classified as forest areas and city areas to carry out experiments for testing the effectiveness of the proposed method.

\subsection{Test results}

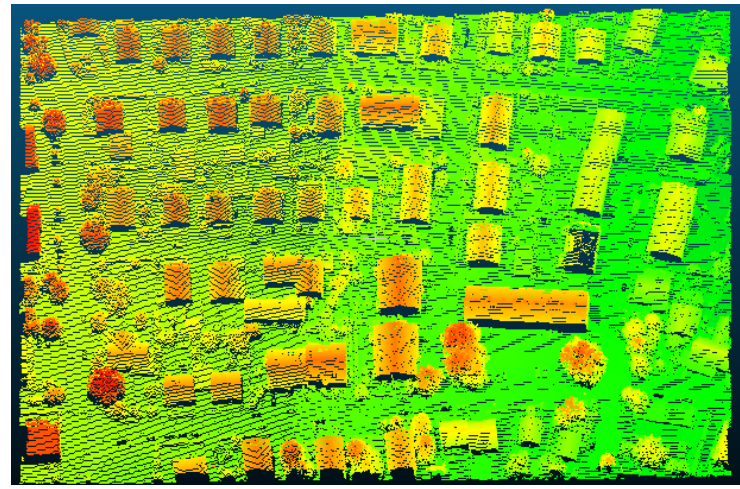

Figure 3.The test data of Vaihingen 


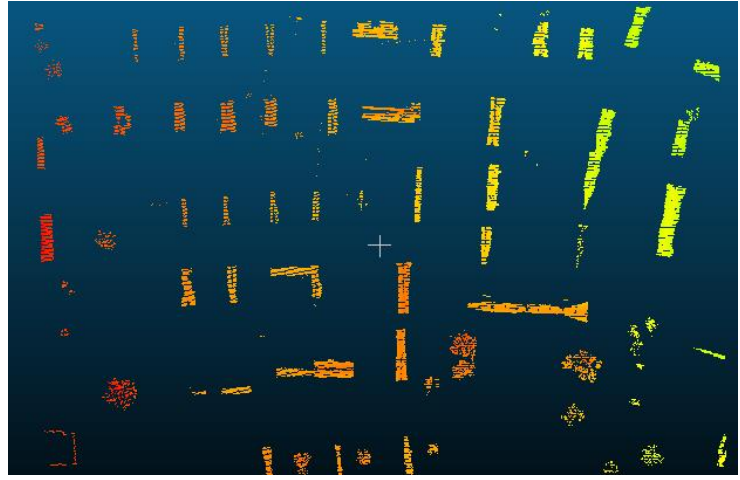

Figure 4.The non-ground seed points in the measured area extracted by the CSF

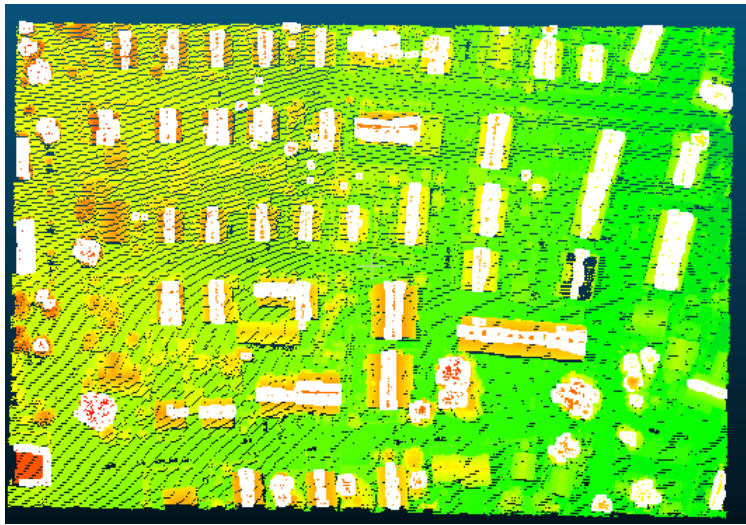

Figure 5.The non-ground point in the measured area filtered by the CSF(superimposed on source data)

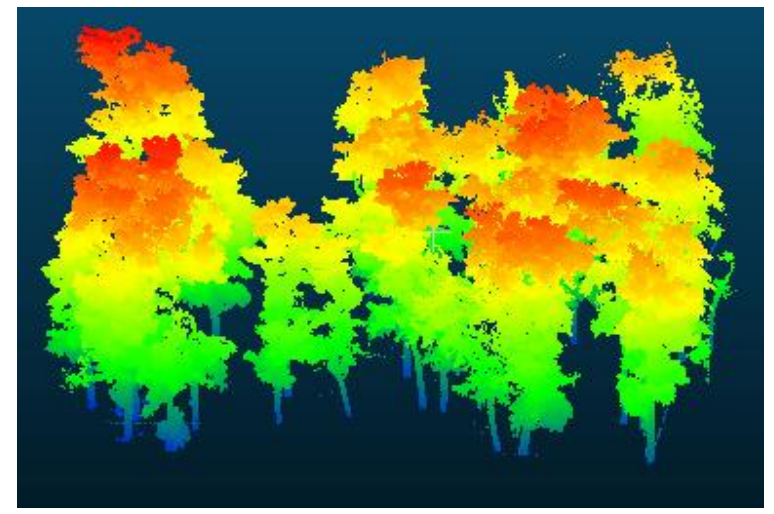

Figure 6.The test data of Yongding River

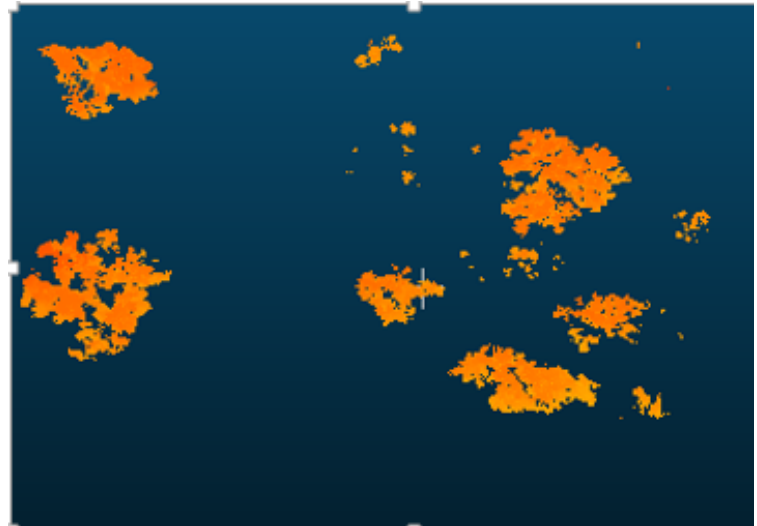

Figure7.The non-ground seed points in the measured area extracted by the CSF

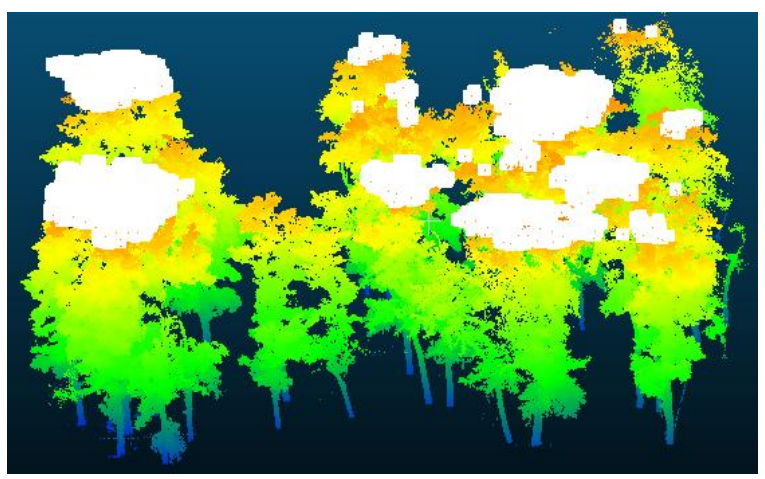

Figure 8 .The non-ground point in the measured area filtered by the $\mathrm{CSF}$ (superimposed on source data)

From the experimental results, it can be seen that the non-ground seed points extracted by CSF are almost all belonged to the filtered non-ground point, which shows that this method can get a group of non-ground seed points and avoid the blind selection of seed points. But if the test area has adjacent objects with largely different height, low objects may not be covered or can't be completely covered, which will lead to the missing of some seed points as the experiment in the forest sample area(there are some low vegetation seed points that are missed).

Wuming Zhang's research pointed out that it is important to choose the right cloth resolution according to the different regions to achieve a good filtering effect. Therefore, for areas where there is a large difference in the local, the softer cloth should be selected so that all non-ground seed points can be extracted. The softness of the cloth can be adjusted by the cloth resolution and the lower the resolution, the weaker the cloth. 


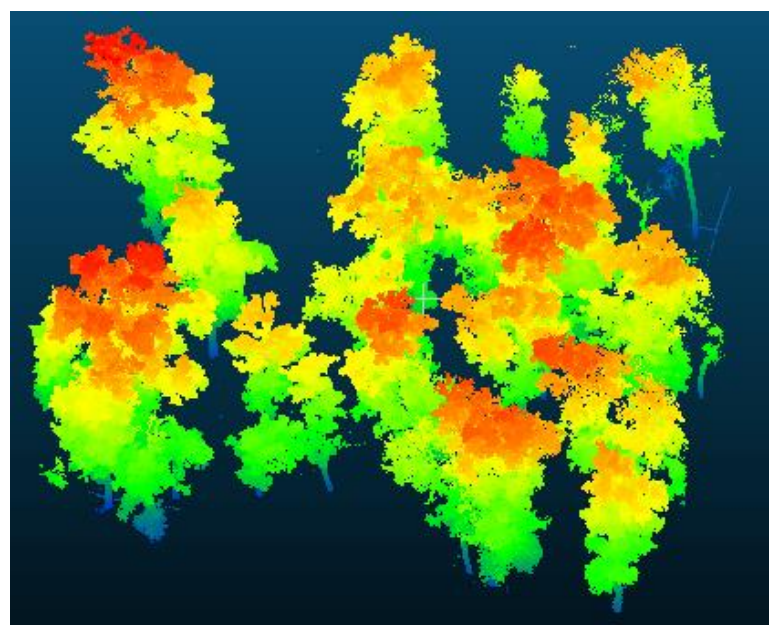

Figure 9.The test data of Yongding River

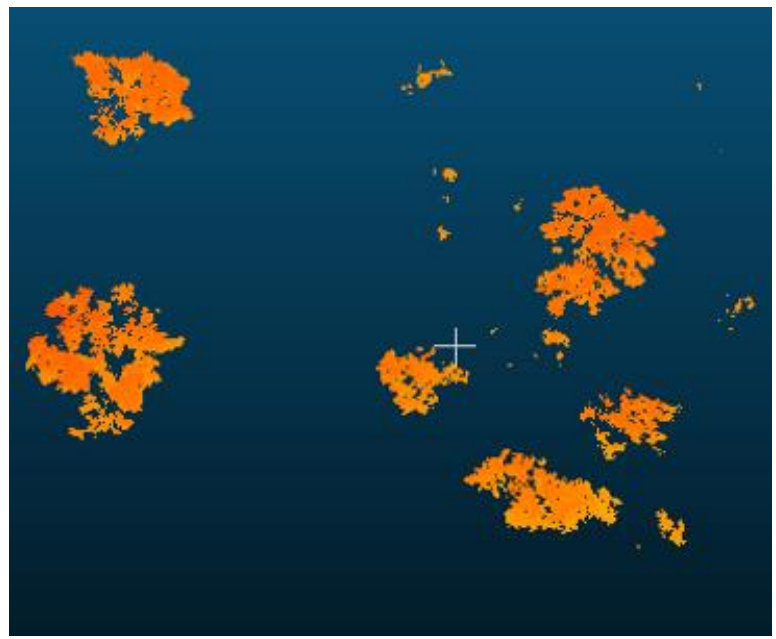

Figure 10.The extraction of non-ground seed points using CSF in the measured area by setting cloth resolution of 2

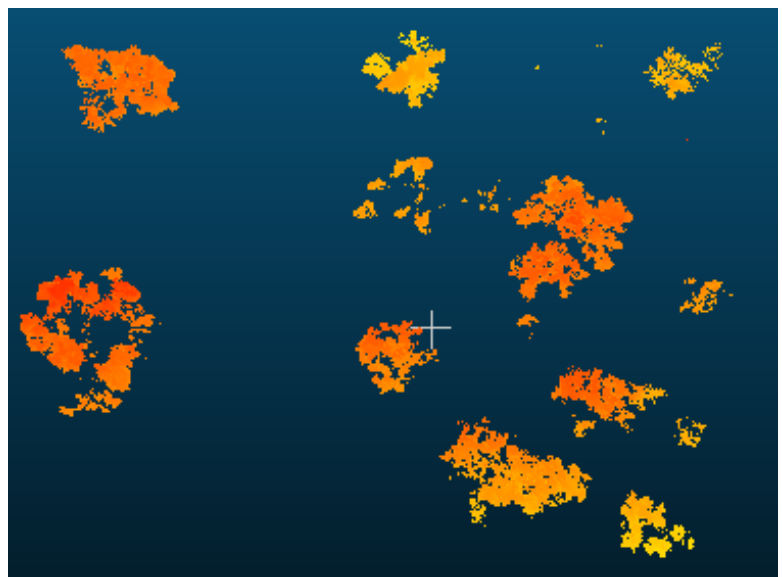

Figure 11.The extraction of non-ground seed points using CSF in the measured area by setting cloth resolution of 1

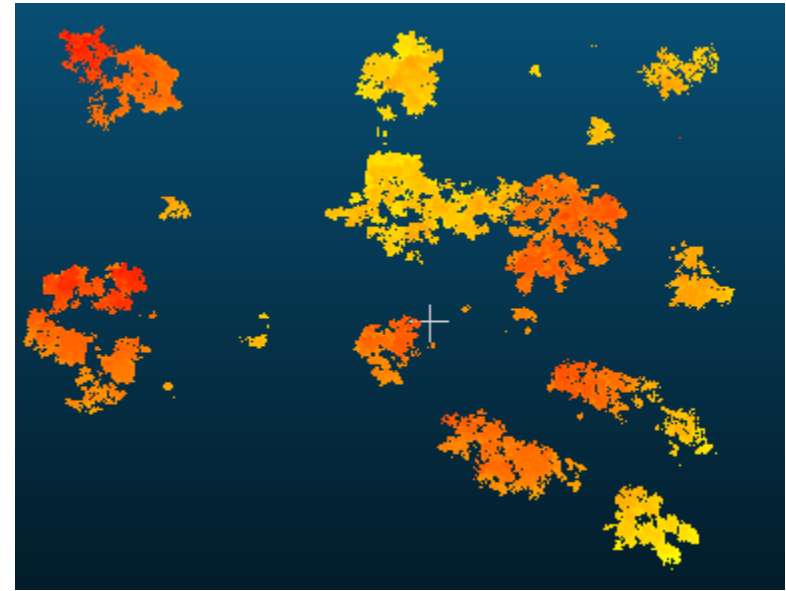

Figure 12.The extraction of non-ground seed points using CSF

in the measured area by setting cloth resolution of 0.5

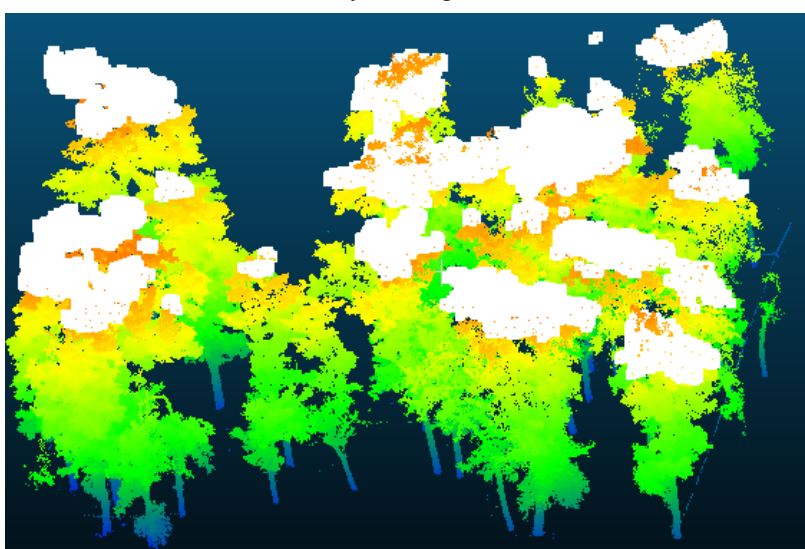

Figure 13.The extraction of non-ground seed points

superimposed on source data(cloth resolution is 0.5 )

It can be observed from the experiment that with the decrease of cloth resolution(ie, represents a softer cloth), the original lowlying vegetation seed points appear, which also shows that CSF has the potential for the extraction of non-ground seed points is low compared to other points. A similar experiment was done on another forest sample area (cloth resolution was set as 0.5 ).

It can be observed that better vegetation seed points can be extracted by the help of CSF. According to the experimental results, CSF filter can be used to extract non-ground seed points with a good representation and can get a group of non-ground point cloud, avoiding the traditional approach which gets a random seed point or only gets isolated seed points. 


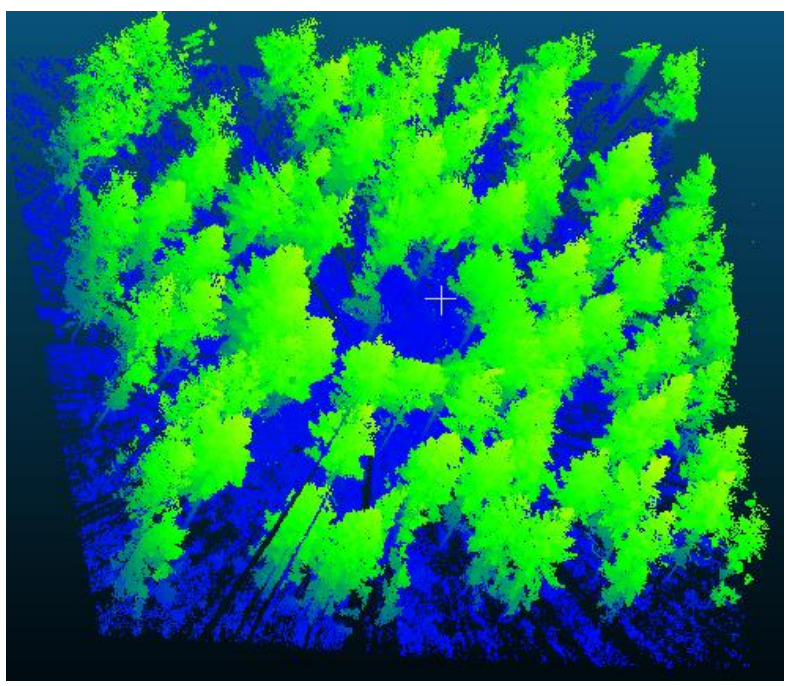

Figure 14.The test data of Chengde

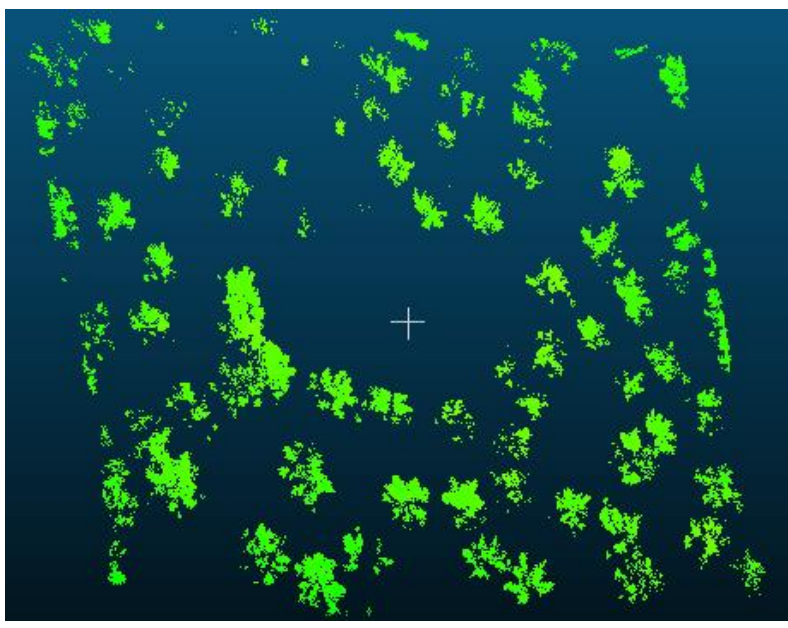

Figure 15.The extraction of non-ground seed points using CSF in the measured area by setting cloth resolution of 0.5

\section{CONCLUSION}

This paper proposes a new method to extract seed points of non-ground points by CSF. The experimental results show that this method can extract a kind of seed points in groups and avoid the blind selection of seed points and the case of isolated seeds only. In order to make full use of these seed points and to achieve region growing results with good robustness, the extracted non-ground seed points can be classified to obtain the seed points of building and vegetation and other seed points. The classified seed points can be used for urban 3D reconstruction as well as vegetation coverage investigation, etc. The research on the classification of seed points after extraction of non-ground points by CSF will be developed in the future.

\section{ACKNOWLEDGEMENTS}

The work was supported by the National Key Research and Development Program of China (NO.2016YFB0501404) and the National Natural Science Foundation of China Grant (Nos. 41331171, 41671414 and 41171265). This work was also supported by the National Basic Research Program of China (973 Program) Grant No. 2013CB733402.

\section{REFERENCES}

Hui Wang, J., 2016. 图像的区域分割与研究. Electronic Technology \& Software Engineering, (5), pp. 99-99.

Jianfeng Lu, J., 2005. Adaptive region growing algorithm in medical images segmentation. Journal of computer-aided design \& computer graphics, 17(10), pp. 2168-2173.

Lu X, J., 2014 The study and application of the improved region growing algorithm for liver segmentation. Optik - International Journal for Light and Electron Optics, 125(9), pp. 2142-2147.

Rook M, C., 2016 Towards automatic semantic labelling of 3D city models. Isprs Ann. Photogramm. Remote Sens. Spatial Inf. Sci.

Sun S,J., 2013. Aerial 3D Building Detection and Modeling From Airborne LiDAR Point Clouds. IEEE Journal of Selected Topics in Applied Earth Observations \& Remote Sensing, 6(3), pp. 1440-1449.

Vo A V, J., 2015 Octree-based region growing for point cloud segmentation. Isprs Journal of Photogrammetry \& Remote Sensing, 104, pp. 88-100.

Wang Y, J., 2016. Three-Dimensional Reconstruction of Building Roofs from Airborne LiDAR Data Based on a Layer Connection and Smoothness Strategy. Remote Sensing, 8(415), pp. 1-24.

Xiuxia Li, J., 2016. Seed extraction method for seeded region growing based on one-dimensional spectral differences. Journal of image and graphics, 21(9), pp. 1256-1264.

Zhengping Hu, J., 2007. Adaptive and high accurate region growing image segmentation method based on region evolution. Systems engineering and electronics, 29(6), pp. 854-857. Zhang W, J., 2016 An Easy-to-Use Airborne LiDAR Data Filtering Method Based on Cloth Simulation. Remote Sensing, 8(6), pp. 501. 